\title{
Las estrategias de aprendizaje en la Educación formal: enseñar a pensar y sobre el pensar
}

\author{
CARLOS MONEREO \\ Universidad Autónoma de Barcelona
}

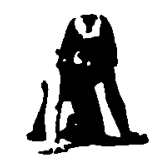

Resumen

En el presente trabajo se analiza la relación existente entre el concepto de estrategias de aprendizaje y de habilidades metacognitivas en el marco de un modelo explicativo basado en la mediación cognitiva. Posteriormente se revisan los métodos instruccionales que se han mostrado más eficaces en la instauración de macroestrategias entre los estudiantes, y se describe de manera pormenorizada un sistema de autointerrogación metacognitiva, el procedimiento metacognitivo de enseñanza-aprendizaje (promete-a). La aplicación de promete-a, en un estudio piloto, a 16 profesores de distintos niveles educativos no-universitarios, ba demostrado tener una incidencia positiva en la planificación, monitorización y evaluación de los procesos cognitivos implicados en la tarea de preparación de sesiones de clase.

Palabras clave: Estrategias de aprendizaje, Habilidades metacognitivas, Métodos instruccionales.

\section{Learning strategies in formal education: Teaching to think and about thinking}

\section{Abstract}

This study analyses the relationship between the concept *learning strategies* and *metacognitive habilities* within the framework of an explanatory model based in cognitive mediation. Later, instructional methods found to be most effective in teaching macrostrategies to students are reviewed. In addition, a metacognitive self-inquiring system is described in detail, i.e., a metacognitive teaching-learning process (Promete-a). The use of Promete-a in a pilot study with 18 teachers from different non-university educational levels showed that this procedure had a positive effect on the planning, monitoring, and assessment of cognitive processes involved in teaching.

Keywords: Learning Strategies, Metacognitives Skills, Instructionals methods.

Dirección del autor: Dpto. de Psicología de la Educación. Universidad Autónoma de Barcelona. 08071 Barcelona.

Original recibido: Septiembre, 1989. Revisión recibida: Abril, 1990. Aceptado: Mayo, 1990 


\section{INTRODUCCION}

Cuando preguntamos a nuestros estudiantes por el tiempo que invertirán en regresar a sus casas al término de las clases, las respuestas suelen ser sorprendentemente precisas, y moduladas en relación a distintas variables (medio de transporte, día y hora, acontecimientos coyunturales, etc.) que los sujetos expresan con gran exactitud. Sin embargo cuando el interrogante se refiere al número de veces que deberán repetir un vocablo desconocido con el fin de retenerlo, o precisar el tiempo que habrán de emplear para «aprender» el contenido de un texto, o describir el sistema o la «táctica» utilizada para recordar una lista de palabras sobre un tema determinado, la mayoría de estudiantes se muestran imprecisos, cuando no perplejos, y en muy pocos casos son capaces de matizar sus respuestas a tenor de las características de la tarea o de variables personales y/o situacionales.

Los principios del mundo físico exterior, parecer ser más conocidos que los principios que rigen nuestros propios mecanismos de conocimiento y aprendizaje, a pesar de que estos últimos son los responsables del dominio de los primeros.

En estos momentos existe un número suficiente de investigaciones que permiten afirmar la importancia crucial que tiene para el aprendizaje el conocimiento y la aplicación eficaz de «estrategias deliberadas» —en la primera acepción introducida en 1960 por Miller, Galanter y Pribram-, para enfrentarse con mayores posibilidades de éxito a situaciones de aprendizaje.

Acogiéndonos a diversas formulaciones (Kirby, 1984; Nisbet y Schucksmith, 1986; Nickerson et al., 1987) podriamos definir las estrategias de aprendizaje (a partir de ahora E/A) como comportamientos planificados que seleccionan y organizan mecanismos cognitivos, afectivos y motóricos con el fin de enfrentarse a situaciones-problema, globales o específicas, de aprendizaje.

Estas estrategias pues, son las responsables de una función primordial en todo proceso de aprendizaje, facilitar la «asimilación» de la información que llega del exterior al sistema cognitivo del sujeto, lo cual supone gestionar y monitorizar la entrada, etiquetación-categorización, almacenamiento, recuperación y salida de los datos.

El papel preeminente que desempeñan estas habilidades no ha tenido suficiente eco en la Educación Formal que sólo de manera limitada ha incorporado una parte de su enseñanza al curriculum escolar bajo el eufemismo de "Enseñar a aprender", con las denominadas habilidades de estudio (sistemas, técnicas, métodos de estudio) que representan el apartado más mecánico y conductual de las E/A, y que sin la participación de habilidades ejecutivas de orden superior, se muestran muy poco transferibles a tareas distintas de las inicialmente ensayadas. A esta crítica a la visión tradicional y restringida de las E/A, imperante hoy por hoy en nuestro país, debe añadirse su orientación a satisfacer las exigencias de las pruebas y exámenes escolares, por encima del objetivo primordial de aprender a aprender, y su tardía introducción, a los 16-18 años, una vez que el alumno ya posee un cierto repertorio de hábitos de aprendizaje, difícilmente modificable (Marland, 1981; Tabberes y Allman, 1983; Cloete y Schochet, 1986; Selmes, 1988).

La necesidad de un nuevo enfoque basado en la enseñanza de E/A de 
orden superior, con un mayor poder de generalización, ha propiciado el inicio de una nueva corriente bautizada, en clara contraposición con el enfoque anterior, como «Enseñar a pensar». Quizás sea Tama (1986) quien ha contribuido a una mejor clarificación de esta vertiente de las E/A, identificando 3 áreas de atención educativa:

1) Un área centrada propiamente en el Enseñar a pensar donde el interés máximo reside en implantar y desarrollar en los estudiantes un conjunto de habilidades cognitivas que les permitan optimizar sus procesos de razonamiento.

2) Una vertiente capitalizada por el Enseñar sobre el pensar, en la que se anima a los alumnos a tomar conciencia de sus propios procesos y estrategias mentales (metacognición), para de esta forma poder controlarlos y modificarlos, mejorando el rendimiento y eficacia en el aprendizaje individual, y por extensión en cualquier tarea de tipo intelectual.

3) Una última perspectiva que podríamos denominar el Enseñar sobre la base del pensar, y que se ocupa de incorporar objetivos de aprendizaje relativos a las habilidades cognitivas, dentro del curriculum escolar, adaptándolas a las distintas áreas de contenido y a los diferentes niveles educativos.

Aun cuando la aportación básica de este trabajo se circunscribe al segundo bloque, la enseñanza de las habilidades metacognitivas, trataremos en primer lugar de delimitar el marco teórico en que nos movemos.

\section{EL APRENDIZAJE COMO PROCESO DE MEDIACION IDIOSINCRASICA}

Desde el momento de su nacimiento, el contacto del niño con el entorno no se produce de forma directa, sino que existen agentes que manipulan, seleccionan, filtran, en definitiva "mediatizan» este contacto, dando forma al contexto (in-formando) a partir de premisas culturales. En este sentido la mediación sería un proceso de trasvase de información de un sistema de representación, caracterizado por un número de bites, una estructura informativa y un código dominante (icónito, gráfico, verbal, motóricogestual) específicos, a otro sistema de representación, mediante la manipulación cognitiva «activa» de dicha información.

Esta mediación se produce en primer lugar fuera del aprendiz, a través de la acción del agente cultural, quien resume, valora, explica, interpreta, amplía o censura la información original, y posteriormente este comportamiento va inferiorizándose, formando sistemas de mediación cognitiva que tienen como misión propiciar el aprendizaje, y a los que nosotros nos referiremos con el término de estrategias de aprendizaje. Este doble proceso de aparición de las funciones psicológicas, primero fuera del sujeto, a un nivel de interacción social, y posteriormente en el interior del sujeto, en el plano mental, tan bien descrito por Vygotski a través de su noción de ley de la doble función, subraya el decisivo componente interpersonal que tie- ne el aprendizaje, y por extensión las estrategias de aprendizaje (ver en esta línea Belmont, 1989).

En la Figura 1 se expone gráficamente dicho proceso de mediación que 
se inicia con la acción de los agentes culturales -padres, maestros, adultos- (mediadores externos) y prosigue con su internalización a través de las estrategias de aprendizaje (mediadores internos).

FiguRA 1

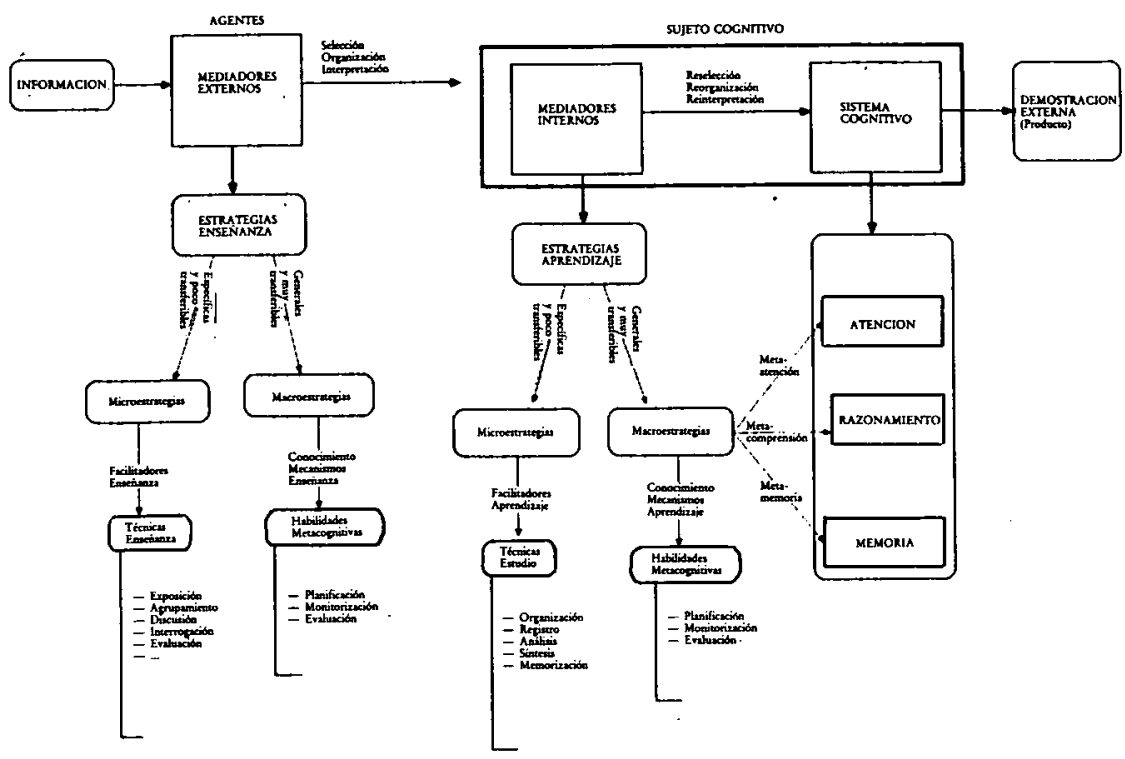

Procesos de mediación en el aprendizaje

Cuando el profesor trata de transmitir unos contenidos a un grupo-clase, no sólo está instruyendo sobre el qué de la materia en cuestión (conceptos, procedimientos, principios y/o valores), sino que simultáneamente está mostrando el cómo tratar y presentar/representar este contenido para ser aprendido. En el momento en que el alumno, en una situación de aprendizaje individual, deba enfrentarse a una tarea, muy probablemente tratará y representará la información para «autoenseñársela» de forma similar a como procede su profesor.

Continuando con este hilo argumental puede afirmarse que el discente que tiene un profesor con un estilo docente directivo, que emplea una metodología expositiva, enfática y repetitiva, basada sustancialmente en el conocimiento de hechos y en la obtención de resultados o productos finales, favorece la interiorización de un tipo de E/A de carácter pasivo y mecánico, en la que más que buscar relaciones significativas entre las ideas, se tiende a efectuar asociaciones y jerarquías arbitrarias en forma de listas (alfabéticas, cronológicas, topográficas, de magnitud, etc.), en la línea de lo que Selmes (1988) denomina «enfoque de aprendizaje superficial».

Contrariamente, cuando el profesor subraya los vínculos relevantes que existen entre las ideas, emplea una enseñanza por descubrimiento en la que exige del alumnado un esfuerzo de comprensión apropiado a su capacidad y próximo a sus conocimientos previos, y trata de incentivar la correcta elaboración de los procesos de resolución, estará favoreciendo la interiorización de E/A de cariz activo y comprensivo, un enfoque de aprendizaje pro- 
fundo, en el que se primará la significatividad de las relaciones entre los elementos del contenido a través de sistemas de representación más próximos a la estructura de la materia en cuestión (redes semánticas, mapas conceptuales, diagramas de flujo, modelos, guiones), y lo que para nosotros es más importante, sustituirá el intento de reproducir las formas de interpretación del profesor como ocurría en el primer supuesto - la «voz de su amo»-, por una interpretación más personal e idiosincrásica de la información. En la medida en que aprender implica «dar significado», todo aprendizaje profundo y duradero requiere una visión personal del sujeto que aprende (Antonijevic y Chadwick, 1982).

Esta correlación entre aprendizaje profundo y aprendizaje idiosincrásico ha sido defendida por diversos autores, aunque desde centros de interés bien distintos. Nos parecen especialmente interesantes las aportaciones de Schank (1988) y de Biggs y Collis (1982), ambos próximos al modelo de procesamiento de la información.

Schank (1988) distingue 3 niveles de comprensión de una misma información: un primer nivel consistente en "dar sentido" que supone la capacidad de explicar con otras palabras, parafrasear, la información; un segundo nivel que denomina de "comprensión cognitiva», en el que el sujeto extrae la estructura o regla que subyace a la información y puede aplicarla a ejemplos paralelos; y un tercer nivel, que correspondería a un aprendizaje profundo, en el que el sujeto añade experiencias y emociones de carácter personal, que promueve la generación de nueva información, y al que el autor llama "comprensión empática».

Biggs y Collis (1982) por su parte, tratan de identificar de qué forma está estructurada la información una vez aprendida y su relación con la comprensión, mediante el análisis de las respuestas que se producen ante preguntas relativas a un material pre-aprendido. Utilizando este sistema elaboran una taxonomía de "la estructura del resultado del aprendizaje» (solo) con cinco tipos de respuesta clasificadas en orden creciente, según el grado de comprensión de lo aprendido que demuestran. Las tres primeras categorias recogen aquellas respuestas que implican únicamente la búsqueda y selección de datos, y su presentación de forma esencialmente descriptiva: Niveles preestructural, uniestructural y multiestructural. Esta clase de respuestas parece organizarse y presentarse de forma similar en todos los sujetos que las emiten y guardan asimismo un enorme parecido a como fue organizada y presentada la información por el emisor originario (profesor, libro de texto, apuntes de un compañero). Se trata pues de un tipo de respuesta general e impersonal, que podría describirse claramente dentro de un enfoque de aprendizaje superficial, explicado con anterioridad.

En el segundo grupo de respuestas, nivel relacionante y de abstracción extendida, los datos aparecen interrelacionados, existe una mayor coherencia entre los apartados, se avanzan conclusiones y se generan dudas e interrogantes pertinentes, fruto del análisis y contrastación con experiencias personales. Estas respuestas, singulares y personalizadas, pertenecen a un enfoque de aprendizaje más profundo, en el que se prima un acercamiento propio, idiosincrásico, a cada problema o tarea.

Dentro del encadenamiento de procesos de Enseñanza-aprendizaje que exige cualquier situación instruccional, desde que el profesor aprende un contenido para enseñarlo, y decide presentarlo de una determinada manera 
(probablemente de forma similar a como él se la ha «autoenseñado»), hasta que el alumno recibe la información y trata de vincularla, con mayor o menor acierto, a sus conocimientos previos - la perspectiva ausubeliana-, organizándola en su memoria de manera más o menos comprensiva, las E/A actúan como eslabones que determinan la cualidad de cada fase. El espectro de las $\mathrm{E} / \mathrm{A}$ acoge desde las mnemotécnicas más rudimentarias, como puede ser la repetición sucesiva de una cifra para su futuro recuerdo, hasta la planificación y supervisión cuidadosa de una acción cognitiva ajustada a las exigencias de una tarea a largo plazo. Esta amplitud de situaciones ha llevado a distintos expertos a tratar de establecer algunas clasificaciones entre las E/A: Estrategias Generales y mediacionales (Resnick y Beck, 1976); ejecutivas y no-ejecutivas (Sternberg, 1983), de procesamiento y de ejecución (Chadwick, 1988); microestrategias y macroestrategias (Kirby, 1984); estrategias de repetición, de elaboración, de organización, de regulación y afectivas (Weinstein y Mayer, 1986). Estas dos últimas aportaciones, desde nuestro punto de vista las más descriptivas y clarificadoras, son las que adoptaremos a partir de ahora.

\section{MICROESTRATEGIAS Y MACROESTRATEGIAS DE ENSEÑANZA-APRENDIZAJE}

A pesar de la dificultad que supone unificar algún criterio categorizador que posea legitimidad científica para agrupar el amplio espectro que conforman las E/A, Nisbet y Schucksmith (1987) han propuesto tres variables que pueden ayudar a delimitar distintas clases de estrategias: 1) La naturaleza del objeto sobre el que incide su acción mediadora-facilitadora; 2) el grado de transferencia a situaciones de aprendizaje diversas que demuestran poseer, y 3) su dificultad para ser enseñadas en marcos instruccionales.

Atendiendo a estos requisitos, Kirby (1984) distingue entre microestrategias de aprendizaje, que actúan entre un problema o tarea específicos y su adquisición por el sistema cognoscente, con un nivel muy limitado de generalización a otros problemas o tareas nuevos, pero muy susceptibles de ser enseñadas; y las macroestrategias de aprendizaje, cuya acción tiene por objetivo el conocimiento y comprensión de los propios mecanismos de aprendizaje que pone en marcha el sujeto, con un elevado grado de transferencia y de difícil, aunque posible, enseñanza. Weinstein y Mayer (1986) son mucho más precisos y completan esta visión dicotómica con una clasificación múltiple:

I. Estrategias de repetición: Comprenderían las prácticas de registro, copia, repetición y rutinarización de técnicas de estudio básicas, con un grado de control cognitivo mínimo.

II. Estrategias de elaboración. Incluirían aquellas técnicas, métodos y formas de representación de datos que favorecen las conexiones entre los conocimientos previamente aprendidos por el sujeto y los nuevos contenidos. En el seno de este grupo se situarían la toma de notas y apuntes, los esquemas, los resúmenes, los diagramas, los mapas conceptuales, etc. Aquí el nivel de control cognitivo aún sería bajo. 
III. Estrategias de organización. Estarían formadas por el dominio de sistemas de agrupamiento, ordenación y categorización de datos, que permitirian obtener una representación fidedigna de la estructura de la información objeto de enseñanza-aprendizaje. A este grupo pertenecen las competencias relativas al orden temporal, espacial o procedimental de eventos, a la identificación de la estructura textual de un escrito - expositivo, narrativo- o a la representación precisa de un tema según el tipo de contenidos que incorpore -conceptos: redes semánticas; principios: modelos; procedimientos: diagramas de decisión; actitudes/valores: jerarquías. El control cognitivo es superior.

IV. Estrategias de regulación: Este bloque abarcaria la utilización de las habilidades metacognitivas en sus distintas esferas: meta-atención, meta-comprensión, meta-memoria. En este caso el grado de control cognitivo exigido es muy elevado.

V. Estrategias afectivo-motivacionales: Este último grupo incluiría las preferencias cognitivas, instruccionales y ambientales que muestra el alumno en el momento de aprender, y las posibilidades de control que es capaz de ejercer sobre estas variables disposicionales. La utilización estratégica, y por lo tanto consciente y propositiva, del estilo personal de aprendizaje, del estilo motivacional, del enfoque u orientación de estudio, o de la localización del control, correspondería a esta división. En este caso el nivel de control ejercido debe ser máximo.

En el caso del estudiante las microestrategias por antonomasia son los métodos y técnicas de estudio, que integrarian las estrategias de repetición y elaboración. En la Tabla I se expone un listado de las microestrategias más repetidas en la literatura especializada (Ontza, 1980; Rotger, 1981; Brunet, 1983; Ibáñez et al., 1983; Genovard et al., 1985; Bransford y Stein, 1986; Alvarez et al., 1988; Howe, 1988; Pérez y Gifra, 1988).

En cuanto a las macroestrategias, vinculadas a las estrategias de organización, regulación y afectivo-motivacionales, sin duda el concepto subyacente que las define son las habilidades metacognitivas, concepto introducido por Flavell en 1970 a partir de sus investigaciones sobre el conocimiento de los niños con respecto a sus mecanismos de memorización (metamemoria), que posteriormente aplicaría al resto de procesos cognitivos (Flavell, 1976; 1981).

Ciertamente aún no existe una clara delimitación entre lo cognitivo y lo metacognitivo, en sus distintas formas: meta-atención, meta-comprensión, meta-memoria, sin embargo todas ellas están relacionadas con la capacidad que tiene un sujeto de valorar el estado de la información dentro de su propio sistema cognitivo (Peralbo, 1987), así como del efecto que tiene ese conocimiento sobre la actuación de estos procesos (Seoane y Garzón, 1986). Es aquí donde el aprendizaje significativo ausubeliano (Ausubel, 1963) encuentra su "punto de fuerza»; en el instante en que el sujeto es consciente de lo que sabe y, sobre todo, de lo que no sabe con respecto a una información, tiene la posibilidad de elaborar una estructura de enlaces y conexiones entre lo conocido y lo nuevo que le permita alcanzar niveles avanzados de comprensión y, aún más, que le indique el camino a seguir para afianzar determinadas relaciones y completar algunas carencias.

Debemos afirmar, sin embargo, que el conocimiento de los propios mecanismos de pensamiento no asegura, por si mismo, la resolución de tareas 
TABLA I

Microestrategias de aprendizaje

\begin{tabular}{|c|c|c|}
\hline Habilidad & Microestrategia & Técnica asociada \\
\hline \multirow[t]{2}{*}{ Planificar } & $\begin{array}{l}\text { CONTROL DEL } \\
\text { ENTORNO DE } \\
\text { APRENDIZAJE }\end{array}$ & $\begin{array}{l}\text { Control de la fatiga } \\
\text { Control distracción } \\
\text { Organización material }\end{array}$ \\
\hline & CONTROL DEL TIEMPO & $\begin{array}{l}\text { Programación corta } \\
\text { Programación larga }\end{array}$ \\
\hline \multirow[t]{2}{*}{ Registrar } & VELOCIDAD LECTORA & $\begin{array}{l}\text { Pre-lectura } \\
\text { Lectura de rastreo } \\
\text { Lectura de repaso }\end{array}$ \\
\hline & $\begin{array}{l}\text { TOMA DE APUNTES } \\
\text { Y NOTAS }\end{array}$ & $\begin{array}{l}\text { Guías de anotación } \\
\text { Abreviaturas }\end{array}$ \\
\hline \multirow[t]{2}{*}{ Analizar } & SUBRAYADO & $\begin{array}{l}\text { Subrayado lineal } \\
\text { Subrayado estructural } \\
\text { Subrayado realzado }\end{array}$ \\
\hline & ANALISIS DE TEXTOS & Guías de análisis \\
\hline \multirow[t]{4}{*}{ Sintetizar } & ESQUEMA & $\begin{array}{l}\text { Esquema circular } \\
\text { Esquema numérico } \\
\text { Esquema gráfico }\end{array}$ \\
\hline & RESUMEN & $\begin{array}{l}\text { Abstract } \\
\text { Fichero }\end{array}$ \\
\hline & $\begin{array}{l}\text { REPRESENTACION } \\
\text { GRAFICA }\end{array}$ & $\begin{array}{l}\text { Diagramas/Histogramas } \\
\text { Gráficas } \\
\text { Ideogramas } \\
\text { Mandalas } \\
\text { Coordenadas temporales }\end{array}$ \\
\hline & CUADRO SINOPTICO & \\
\hline \multirow[t]{2}{*}{ Memorizar } & MNEMOTECNICAS & $\begin{array}{l}\text { Cadena de imágenes } \\
\text { Numéricas } \\
\text { Topográficas } \\
\text { Acrósticos } \\
\text { Asociación palabras } \\
\text { Palabras-muleta }\end{array}$ \\
\hline & CONTROL DEL OLVIDO & Programación de repasos \\
\hline \multirow[t]{3}{*}{ Demostrar } & $\begin{array}{l}\text { PREPARACION DE } \\
\text { PRUEBAS }\end{array}$ & \\
\hline & $\begin{array}{l}\text { CONTROL DE LA } \\
\text { ANSIEDAD }\end{array}$ & $\begin{array}{l}\text { Autocontrol } \\
\text { Relajación } \\
\text { Desensibilización }\end{array}$ \\
\hline & REDACCION & $\begin{array}{l}\text { Monografias } \\
\text { Trabajos investigación } \\
\text { Memorias e informes }\end{array}$ \\
\hline
\end{tabular}

intelectuales, en cambio existe evidencia de que sujetos que únicamente dominan el uso de técnicas específicas, pueden dar con las soluciones correctas como demostraron Cavanaugh y Borkowski (1980) con niños con un hándicap intelectual. 
Las macroestrategias, basadas en el metaconocimiento, son las encargadas de establecer los parámetros de una tarea, localizar los errores, determinar las tácticas y métodos de intervención más apropiadas, controlar su aplicación y tomar decisiones ulteriores a partir de los resultados obtenidos, sin embargo la resolución directa de la tarea corresponde a las microestrategias, a través de operaciones o procedimientos concretos.

A tenor de lo expuesto podemos hablar de una cierta independencia entre ambos tipos de E/A, que la investigación deberá corroborar con mayor solidez, al tiempo que debe subrayarse su necesaria complementariedad en el momento de instaurar aprendizajes óptimos en el repertorio del alumno.

Hasta aquí nos hemos referido sustancialmente a uno de los extremos del «continuum» instruccional: el aprendizaje. No obstante el sujeto que realiza, intencionadamente, la función de emisor, el educador, también pone en juego un conjunto de recursos cognitivos que tienen como finalidad adecuar la información "en bruto" para conferirle el máximo grado de "comprensibilidad» que asegure un aprendizaje profundo.

Esta primera mediación supone la planificación del material a enseñar, partiendo de una representación cognitiva del alumnado (modelo de alumno), más o menos elaborada, que sea capaz de introducir elementos correctores durante su ejercicio, y de la autoconciencia (metacognición) de los propios conocimientos sobre el tema y de las destrezas vinculadas a su didáctica; de nuevo es preciso apelar a un conjunto de macroestrategias, en esta ocasión esgrimidas por el educador, que deberán articularse a través de técnicas específicas de presentación, trasvase y gestión de información (métodos de enseñanza) en el aula. Ciertamente estas microestrategias son características de su área de aplicación, en este caso la enseñanza, no así las macroestrategias que deben sus propiedades de generalización precisamente a la inespecificidad de su contexto de implantación.

\section{FIGURA 2}

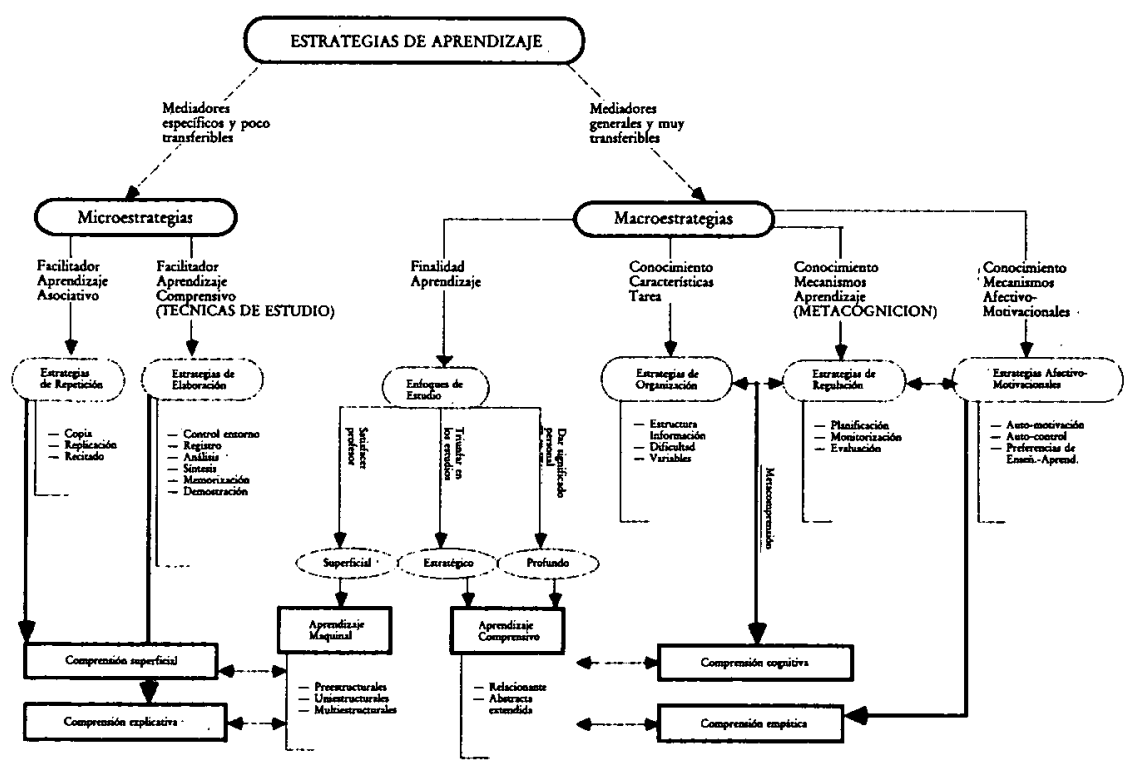

Mapa conceptual sobre las estrategias de aprendizaje 
En la Figura 2 se presenta un mapa conceptual que aporta una síntesis comprensiva de las relaciones conceptuales que sobre las estrategias de aprendizaje hemos tratado de establecer hasta aquí.

Llegados a este punto, $y$ frente al gran número de programas instruccionales que confirman la relativa facilidad de enseñar microestrategias, queda aún la incertidumbre de cómo enseñar macroestrategias.

\section{METODOS DE ENSEÑANZA DE LAS HABILIDADES METACOGNITIVAS}

Dice Main (1985) de forma taxativa: «Los métodos más productivos han sido aquéllos en los que los estudiantes reflexionan críticamente sobre sus propias prácticas en vez de seguir prescripciones de "buenas" prácticas de aprendizaje»; esta máxima expresa con claridad la opinión de los defensores de una enseñanza basada en la promoción de las habilidades metacognitivas, y sólo mediante la explicitación de las propias actuaciones cognitivas, pueden analizarse, discutirse y optimizarse éstas.

Otro autor, Selmes (1988), ha sido aún más preciso en explicar qué principios deben guiar toda enseñanza de $\mathrm{E} / \mathrm{A}$ :

1. Introducir actividades que promuevan la reflexión del alumno sobre sus mecanismos de aprendizaje.

2. Ofrecer la oportunidad de reflexionar y debatir sobre los procesos y tácticas de aprendizaje de otros.

3. Facilitar la aplicación selectiva de las E/A identificadas como eficaces, con prácticas en clase.

4. Meditar sobre los procesos de aprendizaje implicados en tareas de la vida diaria.

5. Apoyar la implantación de las nuevas habilidades y estrategias a lo largo de todas las áreas curriculares.

A pesar de este acuerdo en las intenciones, hoy por hoy existe un número insuficiente de investigaciones publicadas que avalen decididamente la bondad de uno u otro método para la enseñanza eficaz de macroestrategias. El panorama actual se caracteriza por un conjunto de experiencias diseminadas, realizadas con una desigual rigurosidad.

No obstante esta precaria situación, algunos tópicos se repiten con mayor asiduidad y parecen contar con un cierto refrendo de los especialistas en el tema (Nisbet y Schucksmith, 1987, Rubinstein, 1975; Meinchebaum, 1986).

Creemos que esta circunstancia puede justificar la selección de los tres métodos de enseñanza que se describen a continuación.

\section{A) El modelamiento metacognitivo}

El método de modelamiento que propusiera Bandura, consistente en que un modelo refuerza las imitaciones que efectúa un observador de su comportamiento, se toma aquí como punto de referencia pero sustituyendo las conductas observables a imitar por acciones cognitivas que son expresadas verbalmente por el modelo en cuestión, que en cada momento 
hace explícitos los motivos que le llevan a efectuar cada nueva ejecución. El observaddor (alumno), deberá reproducir posteriormente este modo de proceder al enfrentarse con una tarea similar.

Graves (1983) emplea este método en la enseñanza de la escritura. El profesor dentro del aula, al tiempo que va escribiendo una redacción en una transparencia que se proyecta a gran tamaño, expresa en voz alta el proceso cognitivo de resolución que emplea antes, durante y después del ejercicio: establecimiento de objetivos, ordenación de la información, búsqueda de sinónimos, mejora de la expresión, corrección ortográfica, revisiones del proceso, soluciones estilísticas, control de la distracción, valoración del producto final obtenido, etc. Para el autor esta demostración de modelos pierde parte de su eficacia como estrategia cognitiva general si únicamente se emplea de manera episódica, y aconseja su uso generalizado a diversos tipos de escritos.

A esta última sugerencia de Graves fácilmente podríamos añadir la implạntación del modelamiento metacognitivo a otras muchas actividades escolares: lectura, cálculo, problemas matemáticos, manualidades, experimentos de física y química, traducción de idiomas...

\section{B) El análisis y discusión metacognitiva}

El método de análisis y discusión metacognitiva trata de identificar y valorar los procesos de pensamiento que subyacen a un producto o respuesta final, buscando que el alumno sea consciente de la bondad y eficacia de sus propios mecanismos de resolución, y de los de sus compañeros, pudiendo, en su caso, modificarlos.

No se trata pues de "pulir" en lo posible el producto obtenido; posibilidad que en muchas ocasiones, por la baja calidad o incorrección de la estrategia estaría contraindicando, lo que el propio Graves denomina sarcásticamente "hacer la manicura al cadáver», sino de preguntarse sobre qué "he pensado, recordado, imaginado" para llegar a estas conclusiones. El procedimiento suele tener dos variantes: a) el profesor propone una actividad o tarea, y una vez finalizada pide que los participantes escriban o expongan oralmente el proceso cognitivo seguido; b) distribuidos por parejas, algunos alumnos deben resolver una tarea "pensando en voz alta", mientras sus compañeros anotan el proceso cognitivo, para después exponerlo al análisis y discusión de toda la clase.

Este método instruccional ha sido empleado en tareas de memorización de items (Melot, 1987), de lectura individual (Dansereau, 1978; Brown et al., 1979; Lunzer y Dolan, 1979) y en grupo (Larson y Dansereau, 1986).

Un comentario aparte merece el estudio de Clements (1986) sobre las posibilidades del lenguaje informático Logo en el desarrollo de habilidades metacognitivas, mediante el análisis y discusión de las estrategias que el niño emplea para resolver un proyecto o tarea a realizar con el ordenador, tales como decidir la naturaleza del problema, planificar la acción a seguir, escoger los procedimientos más apropiados, comprobar su eficacia y corregir los errores. Flavell (1981) ha insistido en señalar que las experiencias metacognitivas ocurren con mayor frecuencia en situaciones en las que el sujeto debe expresar conscientemente sus razonamientos, y en los problemas que se proponen en Logo el alumno está obligado a reflexionar sobre su 
forma de enfrentarse a la tarea al tener que comunicar, primero a la máquina y después a la clase, el proceso de resolución seguido.

\section{C) La autointerrogación metacognitiva}

El método de autointerrogación metacognitiva tiene un objetivo similar al de las técnicas anteriormente tratadas, conseguir que el alumno conozca las modalidades de procesamiento y decisión cognitivas que emplea con el fin de, ulteriormente, optimizarlas; para ello, y aquí encontramos su peculiaridad, establece un sistema de autorregulación del proceso de pensamiento, a través de interrogantes que el sujeto debe hacerse a sí mismo antes, durante y después de la ejecución de una tarea.

En el procedimiento para su aplicación suelen distinguirse claramente tres fases: a) En primer lugar el profesor propone un modelo de interrogación que emplea en varias tareas-ejemplo. b) Posteriormente cada estudiante pone a prueba el modelo con una gran diversidad de tareas; al principio sugeridas por el docente y después elegidas libremente. c) Por último se intenta que cada discente automatice el procedimiento y sea capaz de utilizarlo de forma independiente.

La autointerrogación metacognitiva, con algunas variantes, ha resultado ser un método eficaz para mejorar el autonocimiento de los propios mecanismos de aprendizaje (meta-aprendizaje) y comprensión (meta-comprensión), como lo demuestran distintos estudios basados en tareas de comprensión lectora.

Tei y Stewart (1985) por ejemplo, establecen un conjunto de interrogantes que el sujeto debe hacerse después de leer un texto. Las cuestiones se refieren a las metas o fines que consideran que persigue la sesión, al reconocimiento de la estructura inherente del texto, a la extracción de la información más relevante del mismo y a la autoevaluación del conocimiento adquirido. Tomlinson (1987), mediante un método similar, incrementa la conciencia de un grupo de estudiantes sobre la presencia o ausencia de «ayudas» a la comprensión (instrucciones, referencias, instigadores...) en un conjunto de textos de distinta complejidad. Más recientemente Cassidy y Baumann (1986) han propuesto también un sistema de generación de preguntas, antes, durante y después de la lectura de textos, como un medio para autocontrolar la comprensión.

En un intento de aunar las diferentes iniciativas que llevan realizándose en este sentido, construimos un instrumento de autointerrogación que, bajo la denominación de Promete-A (Procedimiento Metacognitivo de Enseñanza-Aprendizaje), pudiera ser de utilidad para la planificación, análisis y evaluación de tareas de distinto orden, siempre vinculadas a procesos de enseñanza y/o aprendizaje, en base a la explicitación de las variables metacognitivas intervinientes.

En la Figura 3 se exponen gráficamente las diez etapas de interrogación que incluye Promete-A (Monereo, 1990).

Cada una de estas fases trata de ayudar al sujeto a identificar los principales parámetros que deberá tener en cuenta para resolver la tarea propuesta, y en especial aquellos que se refieren al conocimiento de sus propias modalidades de conocer, aprender y pensar.

Para nosotros es indiscutible que los tres métodos descritos, modelamiento, análisis y autointerrogación metacognitiva, pueden contribuir de 
FIGURA 3

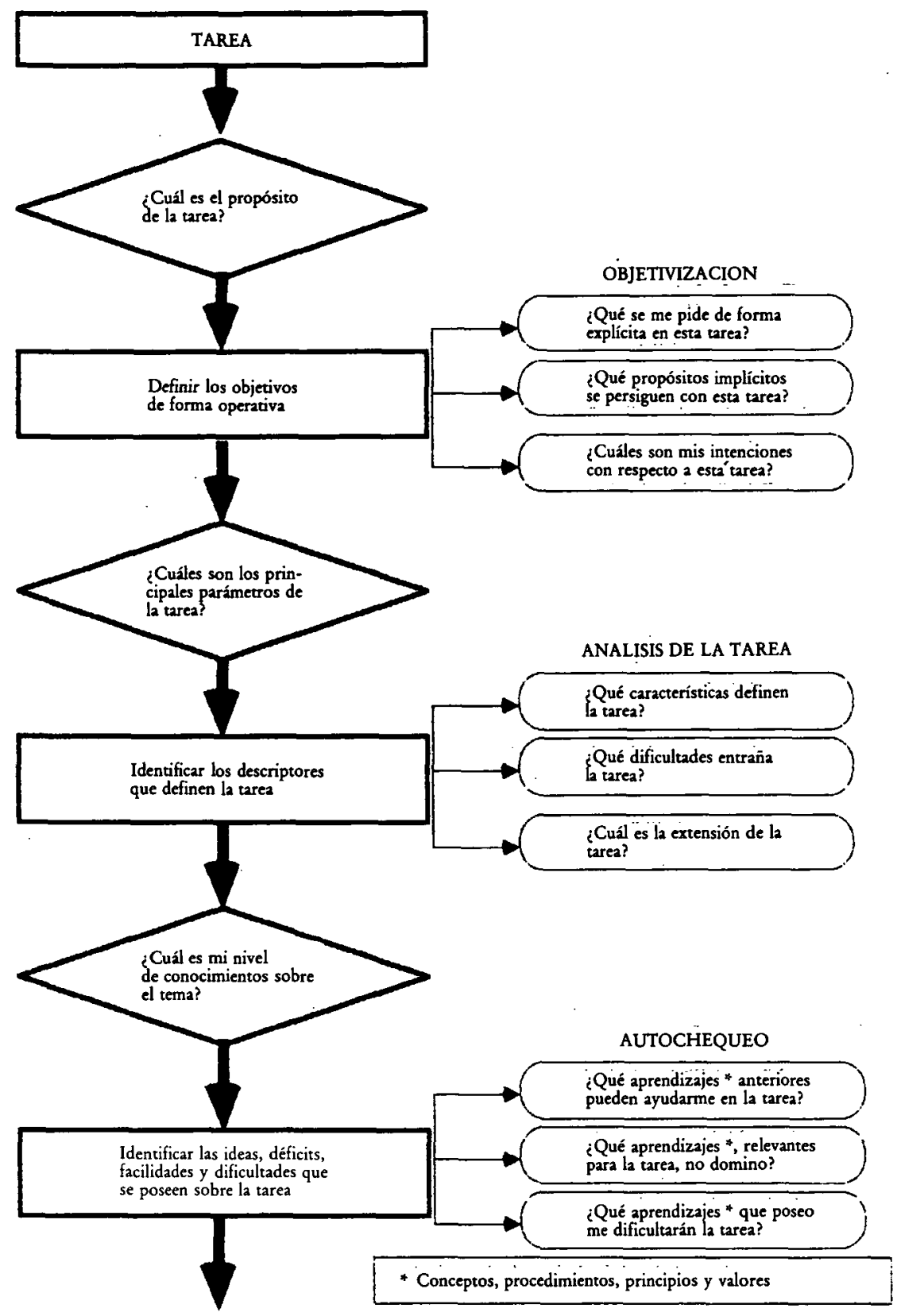

Procedimiento metacognitivo de enseñanza-aprendizaje

manera decisiva no sólo a desarrollar la autonomía de aprendizaje de nuestros estudiantes, sino también a dotarles de sistemas de autoanálisis, autoregulación y auto-optimización de sus propias estrategias cognitivas de enfrentamiento a tareas de enseñanza-aprendizaje.

Del mismo modo, consideramos que la aplicación de estos procedimien- 
FIGURA 3 (Continuación I)

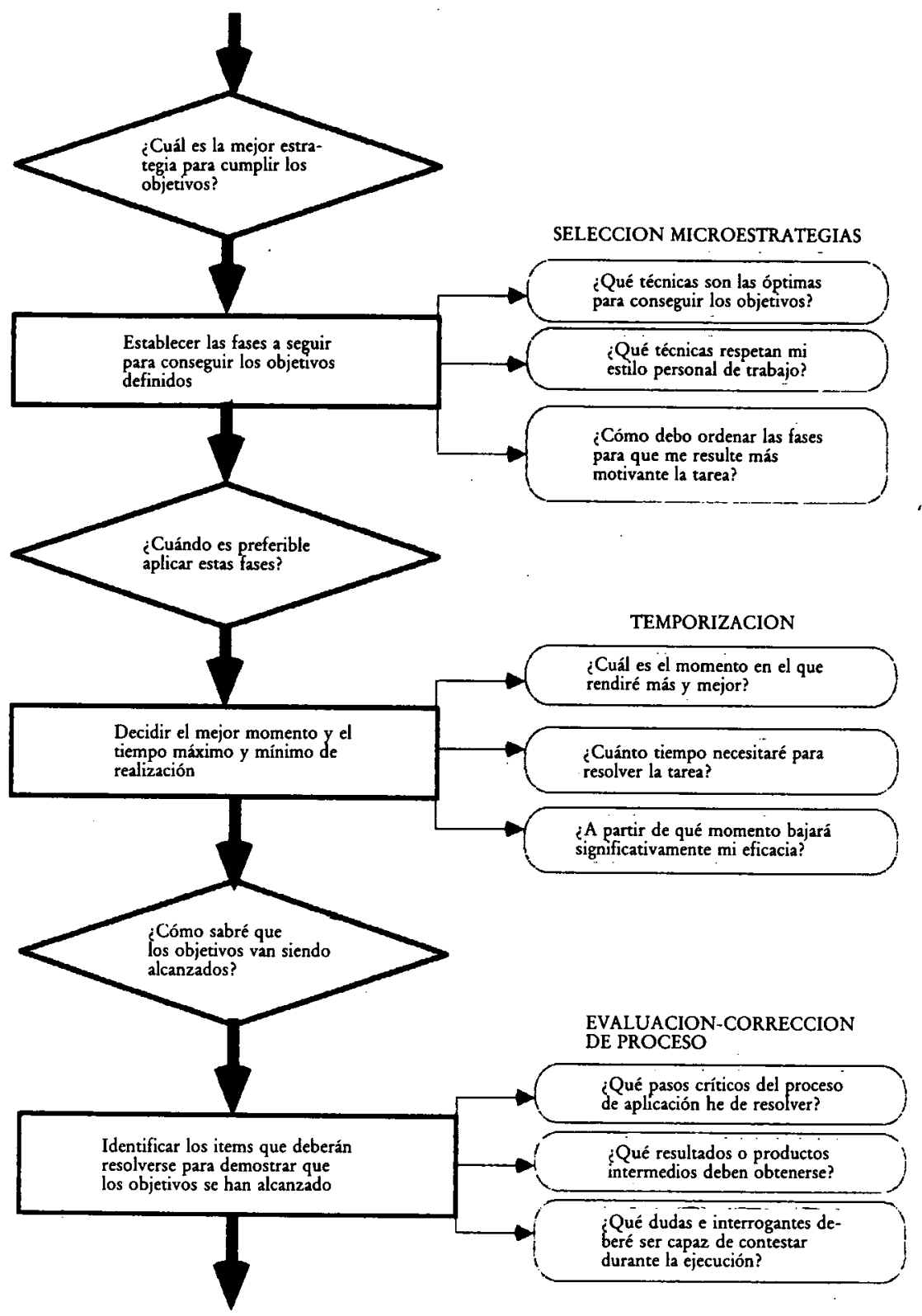

tos al comportamiento docente aportaría elementos de reflexión sobre la propia actuación instruccional, y más específicamente sería un valioso auxiliar en la resolución de situaciones críticas o conflictivas en las que el profesor deba tomar decisiones. Con el fin de efectuar una comprobación previa de las consecuencias que el uso de un procediminto de análisis metacognitivo ocasionaría en la acción docente, decidimos realizar un estudio piloto, que si bien tendría un grado de significatividad y generalización limitados, sí podría proporcionar algunas directrices básicas en vistas a in- 
FIGURA 3 (Continuación II)

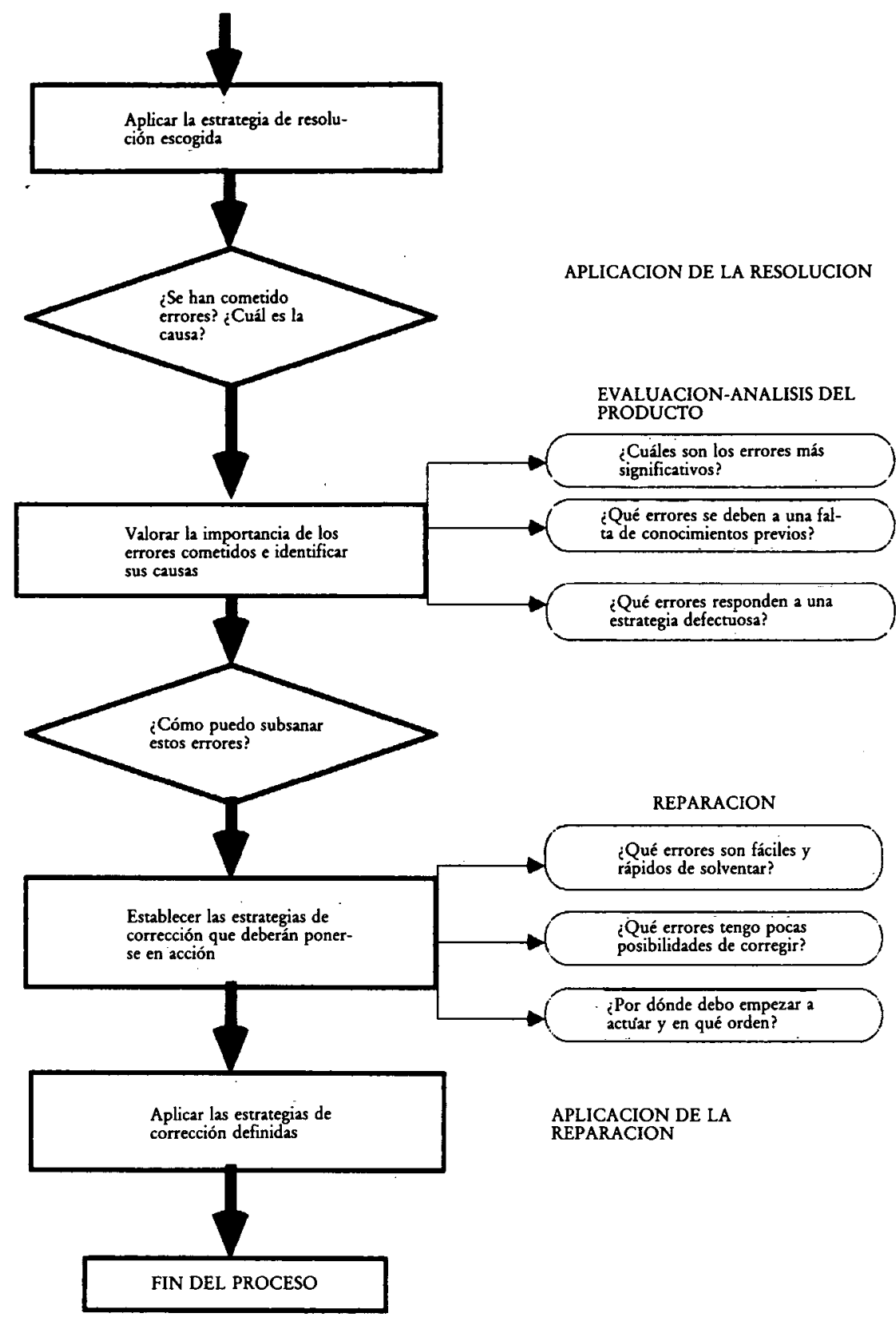

vestigaciones posteriores en las que se estableciese un mayor control de variables.

Persiguiendo este propósito tomamos el diseño, planificación y desarrollo de sesiones instruccionales como variable dependiente, y el procedimiento utilizado para dicha planificación como variable independiente. Nuestra hipótesis era que la utilización de un procedimiento sistemático como Promete-A, que obligase al profesor a reflexionar sobre los distintos compo- 
nentes metacognitivos que influyen de manera decisiva en la toma de decisiones instruccionales (p.e. teorías implícitas y creencias sobre la manera en que aprende el discente, conciencia del propio nivel de conocimientos, conciencia de los propios recursos y déficits metodológicos, etc.) debería ejercer un cambio notable en las distintas fases del proceso instruccional, es decir en la planificación, implantación y evaluación de los aprendizajes a enseñar. A continuación describiremos de manera sistemática los pormenores de este estudio preliminar.

\section{METODO}

\section{Sujetos y muestreo}

En este estudio piloto participaron 18 profesores de ambos sexos de diferentes niveles educativos de Educación Infantil y Primaria pertenecientes a 5 centros privados y 4 centros públicos ( 2 profesores por nivel de $2{ }^{\circ}$ de parvulario hasta $80^{\circ}$ de EGB), cuya edad oscilaba entre los 29 años y los 42 años. Los centros, niveles educativos por centro y profesores (caso de existir más de dos líneas), se escogieron al azar de entre los 52 centros representados por otros tantos estudiantes de segundo ciclo de Psicología (a la vez que profesores de dichos centros) del curso académico 1989-1990, dentro de la asignatura de Psicología de la Instrucción impartida por el autor.

\section{Procedimiento y descripción de las pruebas e instrumentos}

Para dotar a este estudio previo de ciertas garantías de control sobre las variables intervinientes se siguió el siguiente procedimiento:

\section{Primera Fase. Evaluación inicial}

El comportamiento docente de la muestra seleccionada se valoró a partir de una escala de intensidad de 60 items con la siguiente gradación: totalmente de acuerdo, bastante de acuerdo, indeciso, bastante en desacuerdo, totalmente en desacuerdo, a la que se sumó el análisis de una parte de la documentación instruccional: a) el diseño de una unidad didáctica, o en su defecto, las notas o apuntes de preparación de algunas clases sobre el mismo centro de interés; b) el material didáctico de apoyo empleado durante esas clases (esquemas en la pizarra, fotocopias, transparencias, diapositivas, películas de vídeo, maquetas, etc.); c) los apuntes de los alumnos; d) los trabajos realizados en clase y, en su caso, e) los ejercicios, pruebas o exámenes introducidos para valorar el progreso de los alumnos.

Los factores medidos por la escala se centraban en 3 ámbitos, personal, instruccional e interactivo, con un conjunto de factores que describimos someramente a continuación:

1. Variables de ámbito personal

1.1. Motivación hacia la docencia: autosatisfacción en la realización de las tareas docentes o expresión de cansancio, hastío y/o rechazo hacia su ocupación.

1.2. Nivel de actualización en su área de conocimientos: inquietud por 
"estar al día", necesidad de actualización, deseo de sentirse en vanguardia o por el contrario sensación de "arterioesclerosis/aletargamiento/hibernación" científicas.

1.3. Nivel de actualización en la didáctica de su especialidad. Interés por conocer e instaurar en sus clases nuevos métodos de enseñanza-aprendizaje, o en oposición aceptación de un cierto conformismo e inmovilismo didáctico.

1.4. Nivel de estress»: presión psicológica causada por la propia función docente, ya sea por tratarse de una ocupación no deseada o por un sentimiento de incompetencia.

\subsection{Otros factores de tipo personal:}

1.5.1. Dominio de las habilidades comunicativas: competencias relativas a la expresión verbal y gestual puestas en juego durante las sesiones de clase.

1.5.2. Seducción instruccional: capacidad de cautivar el interés de los alumnos y de persuadirlos hacia la ejecución/inhibición de determinadas acciones.

1.5.3. Estilo de enseñanza y de dirección del aula: preferencia por ejercitar el control en las interacciones que se producen dentro del aula (estilo directivo/formal) versus preferencia por otorgar ese control a los propios alumnos (estilo cooperativo/informal).

2. Variables de ámbito instruccional

2.1. Grado de "comprensibilidad" o "enseñabilidad": percepción sobre el grado en que los alumnos aprenden o comprenden sus explicaciones en clase (indicadores: claridad expositiva, capacidad para comunicar ideas, esfuerzo por «darse a entender», nivel de comprensión que facilita, etc.).

2.2. Grado de adaptación al nivel y progreso de los estudiantes: nivel de sensibilidad o adaptabilidad a las necesidades y cambios evidenciados por el alumnado.

2.3. Pertinencia entre los distintos elementos de la programación didáctica: correspondencia entre objetivos de enseñanza-aprendizaje definidos, la secuenciación de los contenidos a instaurar, el tratamiento didáctico elegido para alcanzar dichos objetivos y las modalidades de evaluación seleccionadas para corroborarlo.

\section{Variables de ámbito interactivo}

3.1. Grado de «disponibilidad»: grado de proximidad o distanciamiento del profesor, según la percepción de éste y de sus alumnos.

3.2. Grado de participación: nivel de implicación y de corresponsabilidad del alumno en el desarrollo de las clases.

Importancia que se otorga al intercambio de ideas y experiencias como mecanismo favorecedor de la comprensión.

3.3. Grado de flexibilidad: permeabilidad del profesor a las sugerencias/opiniones de los alumnos. Interés y solicitud ante sus demandas. Nivel de "pasotismo» del profesor con respecto a sus alumnos. 
Segunda Fase. Asignación de los sujetos al Grupo Control (GC) $y$ al Grupo Experimental (GE)

A partir de los resultados de esta evaluación inicial se efectuó la distribución de un componente de cada pareja de profesores, correspondientes a los diferentes niveles educativos mencionados, al GC y al GE, emplazando en este último grupo al par que había obtenido una puntuación más desfavorable.

Tercera Fase. Diseño y desarrollo de las sesiones instruccionales relativas a una unidad didáctica

La actuación del GC consistió en la planificación, desarrollo y evaluación habituales de cinco sesiones instruccionales correspondientes a una unidad didáctica sobre el contenido escolar previsto, mientras que el GE realizó una planificación, desarrollo y evaluación de estas sesiones a partir del Procedimiento Metacognitivo de Enseñanza-Aprendizaje - Promete A- sobre la misma unidad. Las fases, incluidas en la Figura 3 de este artículo, fueron las siguientes:

A) Fase de objetivización: explicitación de los objetivos instruccionales a alcanzar en la unidad didáctica, definición de otros objetivos de aprendizaje, perseguidos de manera colateral (p.e. actitudes positivas hacia el trabajo en grupo), y formulación de otros propósitos o deseos de mayor alcance, no necesariamente vinculados al currículum (p.e. conseguir el respeto de los alumnos).

B) Fase de análisis de la tarea: identificación y comprensión de las características, dificultades y extensión de la unidad didáctica.

C) Fase de autochequeo: reflexión sobre las propias habilidades, recursos y limitaciones relativas tanto a los contenidos involucrados en la unidad didáctica, es decir conceptos, principios, procedimientos y valores a instaurar en el repertorio del alumno, como a la capacidad didáctica para hacerlo efectivo.

D) Fase de selección de microestrategias: elección razonada de la metodología, y de las técnicas instruccionales subyacentes, que se emplearán para conseguir los objetivos definidos en la fase de objetivización, así como del orden en que se introducirán esas técnicas (secuenciación instruccional) atendiendo, por ejemplo, a los propios gustos y preferencias (estrategias auto-motivacionales).

E) Fase de temporizáción: descripción de las preferencias horarias y capacidad personal de productividad en relación al transcurso del tiempo, y por consiguiente previsión de un «timing» en el desarrollo de la unidad didáctica, adaptados a la propia curva de rendimiento personal.

F) Fase de evaluación-corrección de proceso: especificación de los indicadores, tanto de carácter objetivo (p.e. preguntas que deben ser capaces de contestar los alumnos) como de carácter subjetivo (p.e. apreciación del interés de los estudiantes), que deben aparecer durante las sesiones instruccionales para considerar que se van cumpliendo los objetivos o, por el contrario, para introducir sobre la marcha elementos correctores.

G) Fase de aplicación de la resolución: implantación del plan estratégico diseñado. 
H) Fase de evaluación-análisis de producto: identificación y valoración de los fallos cometidos en la planificación y desarrollo de la sesión, y detección de las causas responsables de estos fallos.

I) Fase de reparación: ponderación de la conveniencia y viabilidad de incorporar modificaciones en la sesión instruccional ejecutada y previsión de las acciones de reparación a introducir en una nueva aplicación.

J) Fase de aplicación de la reparación: implantación, en su caso, de la sesión instruccional previamente reparada.

Cuarta Fase. Evaluación «a posteriori» de la incidencia del diseño y desarrollo de las sesiones instruccionales en el comportamiento docente de ambos grupos

Tras la realización de las cinco sesiones consecutivas, se pasó a evaluar las actuaciones docentes aparecidas antes, durante y después de la aplicación de las sesiones instruccionales. Para ello se analizó y comparó, por una parte, el material producido por cada par de profesores durante todo el proceso, y por otra, un autoinforme semiestructurado, con 15 items correspondientes a los ámbitos personal, instruccional e interactivo, elaborado después de cada sesión sobre los aciertos y errores que, subjetivamente, atribuía cada profesor a su propia conducta durante la clase.

\section{Resultados y discusión}

Los resultados obtenidos corroboran, en parte, la hipótesis general apuntada al inicio de este apartado, es decir una incidencia positiva del uso de Promete-A en algunos de los apartados correspondientes a los tres ámbitos considerados del comportamiento docente:

1. Se obtienen diferencias sustanciales entre ambos grupos en la satisfacción expresada por la realización de la tarea (variable: motivación hacia la docencia). Así mientras la totalidad (9 sobre 9) de los profesores de GE, a pesar de las resistencias iniciales relativas al tiempo que exigía el nuevo procedimiento, manifestaron un interés creciente por preparar nuevas sesiones, únicamente dos profesores de GC ( 2 sobre 9 ) constataron cierto progreso en su satisfacción con respecto a la tarea de planificar sesiones instruccionales.

2. En el ámbito instruccional, como era presumible anticipar, es donde Promete-A tiene una mayor influencia, destacando sus efectos sobre 3 subvariables: en la percepción del grado de "comprensibilidad» de los alumnos, en la pertinencia observada de los distintos estadios de la programación de los contenidos y en la aplicación de instrumentos de control del progreso experimentado por el alumnado.

En relación a la primera variable, los 8 profesores del GE comunicaron una positiva y masiva modificación en su capacidad de "darse a entender" y por consiguiente un cambio muy favorable de su percepción de «enseñabilidad» con respecto a su alumnado. En el GC, ningún docente expresó cambios en este sentido.

En el segundo aspecto, 7 profesores del GE muestran un cambio muy positivo en el grado de pertinencia instruccional conseguido en sus Unidades Didácticas, y singularmente en la construcción de secuencias de actividades justificadas en base a criterios psicopedagógicos de una notable tras- 
cendencia. Por contra, sólo un profesor del GC consigue una mayor pertinencia en su programación.

En cuanto a la segunda variable, la introducción sistemática de sistemas de evaluación inicial y procesual, es claramente superior el GE, 8 profesores, frente a un único caso en el GC.

3. Por último en el ámbito interactivo cabe señalar como nota preeminente, la mejora en la flexibilidad o permeabilidad del profesor a las sugerencias de sus estudiantes, en 8 de los docentes del GE, dándose una respuesta similar en 3 profesores del GC.

A estos resultados generales deben añadirse tres comentarios que mayoritariamente aparecieron en los autoinformes de los profesores del GE: la necesidad de proporcionar mayores instrucciones y técnicas de apoyo para paliar las dificultades encontradas en la resolución de la fase de autochequeo, una de las mejor valoradas por el profesorado; la importancia decisiva de explicitar los indicadores de proceso, antes de impartir las clases, y el incentivo que supone preparar una sesión de enseñanza-aprendizaje buscando una ordenación de las distintas secuencias que sea "personalmente motivador".

Al cabo de un mes de efectuado el estudio, y de forma espontánea, 6 de los 9 profesores del GE seguían utilizando Promete-A para preparar la mayoría de sus sesiones instruccionales.

Los datos aquí expuestos son el producto de un estudio piloto cuya finalidad es la preparación de un diseño de investigación de fundamentación experimental, y por lo tanto carecen del rigor metodológico que les otorgaría significatividad, sin embargo consideramos que aportan una base suficientemente sólida sobre la que formular hipótesis, en la línea de los resultados obtenidos, que posean visos de credibilidad.

\section{CONCLUSIONES}

Hasta aquí hemos tratado de enfatizar la importancia que tienen las $E / R$ en el nivel de significatividad en que se produce el aprendizaje, y por consiguiente el interés que tiene su enseñanza en el marco de la Educación Formal. Esta Enseñanza, sin embargo, debería respetar dos directrices básicas que exponemos seguidamente.

En primer lugar que la enseñanza de $E / R$ se vincule a la programación de los contenidos correspondientes a las distintas áreas curriculares, y que tanto las micro como las macroestrategias, el cómo aprender un tema, se introduzcan junto con el qué aprender, la materia en cuestión; Coll (1987) expresa esta idea con acierto cuando afirma: «La adquisición de los procesos y estrategias que subyacen al objetivo de aprender a aprender, no puede contraponerse a la adquisición de otros contenidos".

En segundo lugar, que el profesor responsable de su inclusión en las Unidades Didácticas utilice también algunos procedimientos estratégicos de enseñanza-aprendizaje para analizar y optimizar su comportamiento con respecto a las diferentes tareas relacionadas con su función docente. Todos aquellos métodos que favorezcan a las propias decisiones y ejecuciones instruccionales, están especialmente indicados en la consecución de este propósito. 
Ambos requisitos apuntan al diseño e implantación de Proyectos Curriculares de Centro basados en la $E / R$, y muy especialmente en las habilidades metacognitivas. Frente al «enseñar a aprender» y el «enseñar a pensar», en la Educación Formal deberá imponerse el «enseñar sobre la base del pensar», o lo que es lo mismo enfatizar el aprendizaje del medio como parte sustancial y constitutiva del mensaje para, en última instancia, formar ciudadanos competentes en la gestión de sus propios aprendizajes.

\section{Referencias}

Alvarez, M., et al. (1988). Métodos de estudio. Martinez Roca: Barcelona.

ANTONIJEIC, N. y CHADWICK, C. (1982). Estrategias cognitivas y metacognición. Revista de Tecnologia Educativa, 4. Vol. 7, 307-321.

AusuBEL, D. (1963). The Psychology of Meaningful Verbal Leaming. Nueva York: Grune \& Stratton.

Belmont, J. (1989). Cognitive Strategies and Strategic Learning. The Socio-Instructional Approach. American Psychologist, 2. Vol. 44, 142-148.

BIGGS, J., y CoLLIS, K. (1982). Evaluating the quality of Learning: The SOLO Taxonomy. Nueva York: Academy Press.

BRANSFORD, J., y STEIN, B. (1986). Solución IDEAL de problemas. Barcelona: Labor.

BRown, A., et al. (1979). Training Self-Checking Routines for Estimating Test Readiness: Generalization from list Learning to Prose Recall. Child Development, 50, 501-512.

BRUNET, J. (1983). ¿Cómo programar las técnicas de estudio de EGB? Madrid: San Pío X.

CASSIDY, M., y BAUMANN, J. (1986). How to incorporate comprehension monitoring strategies into basal reader instruction. The Reading Teacher, 40. Vol. 1, 28-31. (Traducción: Cómo incorporar las estrategias de control de la comprensión a la enseñanza con textos base de lectura. Comunicación, Lenguaje y Educación, 1, 1989, 45-50.

ClemENTs, D. (1986). Theoretical Framework for the Development of Metacognitive Abilities in Logo Environments. En Clements, D. Pre-Proceedings. Massachussets: M.I.T., 115-118.

CLOETE, N., y SCHOCHET, I. (1986). Alternatives to the Behavioral Technicist Conception of Study Skills. Higher Education, 15. Vol. 3-4, 247-248.

COLL, C. (1987). Psicología y currículum. Barcelona: Laia.

CHADWICK, C. (1985). Estrategias cognitivas, metacognición y el uso de los microcomputadores en la Educación. Planiuc, 7, 113-129.

DANSEREAU, D. (1978). The Development of a Learning Strategies Curriculum. En O'NEIL, H. Learning Strategies. Nueva York: Academic Press, 1-29.

FlavelL, J. (1970). Developmental Studies of mediated Memory. En REESE, H. y LIPSITT, L. Advances in Child Development and Behavior. Nueva York: Academic Press.

FLAVELL, J. (1976). Metacognitive aspects of Problem solving. En RESNICK, L. The Nature of Intelligence. Hillsdale: Erlbaum.

Flavell, J. (1981). Cognitive Monitoring. En Dickson, W. Children's Oral Comunication Skills. Nueva York: Academic Press.

GenOVARD, C., et al. (1985). Hábitos de estudio. Bellaterra: I.C.E.-U.A.B.

GRAVES, D. (1977). Writing: Teachers and Children at Work. Londres: Heinemann.

Howe, A. (1988). How to study. Londres: Kogan Page. (Traducción: Cómo estudiar. Bilbao: Deusto).

IBÁNEZZ, R., et al. (1983). Eficacia en el estudio. Madrid: Anaya/2.

KIRBY, J. (1984). Cognitive Strategies and Educational Performance. Nueva York: Academic Press.

LARSON, C., y DANSEREAU, D. (1986). Cooperative Learning in Dyads. Journal of Reading, 29. Vol. 6, 516-520.

LUNZER, E., y DOLAN, T. (1979). Reading for Learning in the Secondary School. En AsHER, C. Language, Reading and Learning. Oxford: Basil Blackwell.

MaIN, A. (1985). Reflection and the Development of Learning Skills. En BOUD, D.; KEOGH, R., y WALKER, D. Reflection: Turning Experience into Learning. Londres: Kogan Page.

MARLAND, M. (1981). Information Skills in the Secondary Curriculum. Londres: Methuen.

MELOT, A., y NGUYEN, A. (1987). El conocimiento de los fenómenos psicológicos. En OLERÓN, P. El niño, su saber y su saber hacer. Madrid: Morata, 226-286.

Miller, J.; Galanter, E., y PRIBRAM, K. (1960). Plans and the Structure of Bebavior. Nueva York: Holt.

MONEREO, C. (1990). Macroestrategias de enseñanza: aplicación en la preparación de sesiones de formación. En MONEREO, C. (Comp.). Aprender a aprender y a pensar en la escuela. Madrid: Aprendizaje y COMAP. 
NICKERSON, R., et al. (1987). The Teaching of the Thinking. Nueva Jersey: Lawrence Erlbaum Asso. (Traducción: Enseñar a pensar. Buenos Aires: Paidós).

NisBET, J., y SCHUCKSMITH, J. (1987). Learning Strategies. Londres: Routledge \& Kegan Paul. (Traducción: Estrategias de aprendizaje. Madrid: Santillana).

ONTZA, J. (1984). Saber estudiar. Bilbao: Mensajero.

PÉREZ, M., y GIFRA, A. (1988). Tècniques d'estudi. Vic: Eumo.

RESNICK, L., y BECK, I. (1976). Designing Instruction in Reading: Interaction of Theory and Practice. En GUTHRIE, J. Aspects of Reading Acquisition. Baltimore: Johns Hopkins Univ. Press.

ROTGER, A. (1981). Las técnicas de estudio en los programas escolares. Madrid: Cincel-Kapelusz.

Schank, R. (1988). En Sternberg, R., et al. ¿¿Qué es la inteligencia? Madrid: Pirámide.

SELMES, I. (1988). Improving Study Skills. Londres: Hoder \& Stoughton. (Traducción: La mejora de las habilidades de estudio. Madrid: Paidós).

SeoAne, J., y Garzón, A. (1986). Metacognición. En VegA, J. Diccionario de Psicología de la Educación. Madrid: Anaya, 290-294.

STERNBERG, R. (1983). Criteria for Intellectual Skills Training. Educational Researcher, 12. Vol. 2, 6-12.

TabBeres, R., y Allman, J. (1983). Introducing Study Skills: An Appraisal of Initiatives at $16+$. Windsor: NFER-Nelson.

TAMA, M. (1986). Thinking Skills: A return to the content area classroom. Comunicación presentada en el "Meeting of the International reading Association", Philadelphia, 13-17 de abril de 1986 .

TEI, E., y StEWART, O. (1985). Effective Study from text: Applying Metacognitive Strategies. Forum for Reading, 16. Vol. 2, 46-55.

TOMLINSON, L. (1987). Recognition to recall: Self-Questioning to Enhance Student's Metacognition of Organization and Demands of text. Comunicación presentada en el Annual Meeting of the International Reading Association celebrado del 3 al 7 de mayo de 1987 en Anaheim.

WeINSTEIN, C., y MAYER, R. (1986). The teaching of learning strategies. En WITTROCK, M. (ed.). Handbook of Research on Teaching. Nueva York: Macmillan.

\section{Extended Summary}

The aim of this paper is to establish a direct relationship between three areas of study which are at present receiving great attention in the litterature: cognitive mediation, learning strategies, and metacognitive skills. For this purpose, it was decided to adopt an explanatory model of the learning which results from mediation processes which occur between exogenous and «objective» information from the environment, and information which after undergoing several adjustments and modifications is learned, i.e., information susceptible to be used in different tasks in a "personal" or idiosyncratic way. Following this line of arguments, learning strategies would be systems of cognitive mediation inferred and internalized by the subject after being repeatedly exposed to various forms of information from close cultural agents, basically these are parents and teachers.

These learning strategies are used by the student as real self-instructional methods, especially in situations of individual study. Therefore, the nature of these strategies will determine whether the nature of student learning is of the meaningful-deep or asociative-superficial type.

Following Nisbet and Schucksmith's (1987) criteria, the sole object of these strategies may be to assimilate specific content, such as material related to the curriculum. In this case, we would be within the frame of microstrategies, and more specifically these would be what Weinstein and Mayer (1986) term as repetition and elaboration strategies. On the other hand, a part or the whole of the object of the strategy may be the cognitive mechanism itself which the subjects sets in motion for data processing. In this 
case, the subject would be using organization, regulation, and affective-motivational strategies, which may be grouped under macrostrategies.

As these macrostrategies have priority in the subject's consciousness of his/her own cognitive processing activity, the subject must use a set of metacognitive skills. Their main advantage is that when these skills affect those processess which support the majority of mental actions they have a great capacity for transference. However, their instructional treatment involves great difficulties of specification.

Some of the methods found to be more efective in the instruction of self-regulatory metacognitive skills may be grouped into three separate blocks:

a) Methods based on metacognitive modelling. Experts act as «models of thought" in relation to specific cognitive behaviour which the learner must try to imitate.

b) Metacognitive analysis and discussion methods interested in "a posteriori evaluation of the cognitive processes and metacognitive activities associated to individual behaviour.

c) Methods related to the planning, monitoring, and assessment of the cognitive processes which underlie performance. These are carried out through a self-questioning procedure which the subject undertakes in each of the phases mentioned.

In the paper, the pilot study consisting in the preparation of instruction-learning sessions, carried out by 18 school teachers in different educational levels, is explained. An initial assessment using a questionaire with 60 items relating to 3 areas of teacher behaviour (personal, instructional, and interactive) was carried out. As a result, a control group (CG) was established in which teachers continued to prepare lessons in their usual manner; and an experimental group (EG) where a self-inquiry procedure, developed by the author under the same Promete- $a$, was used to prepare their lessons.

Despite of shortcomings in the design, pertaining to a preliminary study, a substantial improvement was found for the EG over the CG with respect to three aspects: 1$)$ greater relevance of the different phases of instructional programing, particularly in the decision criteria applied in sequencing instruction-learning activities; 2) systematic introduction of initial and final assessment methods; and 3) improved perception on the degree of student understanding of instructional material.

These initial results encouraged us to undertake a larger and more rigorous investigation which is at present under way. 